\title{
LOS RESTOS DE MALASSEZ, VIDA Y DESTINO EN LOS TEJIDOS ODONTOGÉNICOS. UNA REVISIÓN DE LA LITERATURA
}

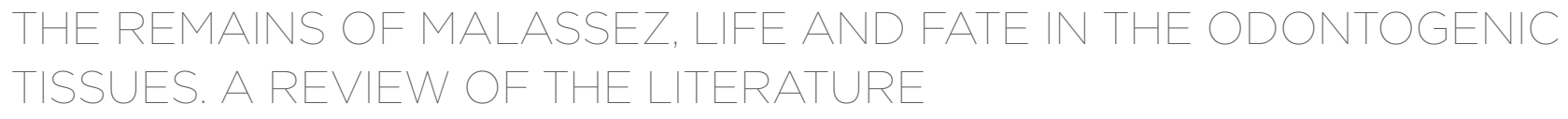

Paola Elena Medina-Ocampo ${ }^{*}$ (i)

paomedinaocampo@gmail.com

\section{Gustavo Adolfo Fiori-Chíncaro ${ }^{2}$ (i)} gfiori@ilaeperu.com

Artículo recibido: 12/02/2020

Arbitrado por pares

Artículo aceptado: 26/03/2020

\section{* Autor corresponsal:}

Paola Elena Medina-Ocampo paomedinaocampo@gmail.com

\section{RESUMEN}

Los restos epiteliales de Malassez son células que se encuentran alrededor de las raíces de las piezas dentarias y forman parte de los tejidos del ligamento periodontal, donde se disponen en forma de red. Las funciones que desempeñan no son muy específicas; sin embargo, se demostró su participación en el mantenimiento del espacio del ligamento periodontal, la regeneración del tejido periodontal, la regeneración del cemento, entre otros. Además, tienen capacidad de diferenciarse en otros linajes celulares, lo que demuestra su capacidad como células madres, y el papel más conocido que tienen es su participación y proliferación en la formación de diversas patologías, como quistes y tumores odontogénicos.

Palabras clave: restos de Malassez, ligamento periodontal, células madre, quistes odontogénicos, tumores odontogénicos

\begin{abstract}
Epithelial rests of Malassez are cells that are arranged in a network located around the roots of the teeth forming part of the periodontal ligament tissues. The functions that these cells perform are not very specific, however, they have shown to participate in the maintenance of the periodontal ligament space, regeneration of periodontal tissue, including cement regeneration among others. In addition, they are able to differentiate into other cell lineages, thereby demonstrating their capacity as stem cells. These cells are best known for their role in the participation and proliferation of the formation of different pathologies, such as cysts and odontogenic tumors.
\end{abstract}

Keywords: Rests of Malassez; Periodontal ligament; Stem cells; Odontogenic Cysts; Odontogenictumors

\section{INTRODUCCIÓN}

El descubrimiento de los restos epiteliales de Malassez se remonta a 1885, cuando el investigador Louis-Charles Malassez encontró estas células en forma de red alrededor de la raíz dentaria, incluida el área de furcación. Estos restos se derivan de los fragmentos de la vaina de la raíz epitelial de Hertwig, que es una estructura transitoria ya que, después de cumplir sus funciones durante el proceso de desarrollo de la raíz, es fenestrada y reducida a lo que se denomina restos epiteliales de Malassez (REM). Estos se localizan en los tejidos del ligamento periodontal como pequeñas agrupaciones que forman islas con una alta relación núcleo-citoplasmática, es decir, el núcleo llega a alcanzar el tamaño del citoplasma y están muy próximas al cemento radicular, por lo que contribuyen a su regeneración y reparación ${ }^{(1-6)}$.

Citar como: Medina-Ocampo PM, FioriChíncaro GA. Los restos de Malassez, vida y destino en los tejidos odontogénicos. Una revisión de la literatura. Rev Cient Odontol (Lima). 2020; 8(1): e008.

DOI: $10.21142 / 2523-2754-0801-2020-008$

\footnotetext{
1 Facultad de Odontología, Universidad Mayor de San Andrés. La Paz, Bolivia.

2 Instituto Latinoamericano de Altos Estudios en Estomatología (ILAE). Lima, Perú.
} 
La función de los REM aún no es muy específica; sin embargo, muchos estudios han demostrado que tienen funciones importantes en cuanto al mantenimiento del diente y el periodonto durante toda la vida. Se ha hallado que tienen participación en la homeostasis del ligamento periodontal y en el mantenimiento del espacio de este para prevenir la anquilosis dentoalveolar, e intervienen además en la formación y reparación de esmalte $\mathrm{y}$ cemento. En cuanto a esto último, se describe que podría darse la diferenciación de cementoblastos mediante la transición epitelial-mesenquimatosa, lo que contribuye al desarrollo de la ingeniería tisular de los dientes y el periodonto $\left({ }^{4,5,7-9}\right)$.

La cercanía de estas células a tejidos de la zona periodontal o periapical puede producir que los restos proliferen y den como resultado la formación de procesos inflamatorios y neoplásicos en el transcurso del desarrollo, pues es durante este periodo que las células presentan una capacidad potencial de formar tejidos al presentarse como numerosas islas de células epiteliales escamosas no queratinizadas, bien diferenciadas $\mathrm{y}$ redondeadas, distribuidas en un tejido fibroso. Es por esta razón que varios autores han descrito su participación en la formación de lesiones patológicas, entre quistes y tumores odontogénicos. Así mismo, estudios recientes han demostrado que cumplen una función importante no solo en el ligamento periodontal normal, sino también en los tratamientos periodontales regenerativos y el mantenimiento del movimiento ortodóntico $(1,3)$.

Además, los estudios han demostrado que los REM son considerados reservorios de células madre epiteliales y embrionarias, con alta capacidad de diferenciación, que se pueden aislar y modificar, lo que permite su cultivo in vitro para la formación posterior de diferentes tejidos como óseo, cemento y estructuras como las fibras de Sharpey $\left({ }^{10-14}\right)$.

También se ha demostrado su capacidad para diferenciarse en ameloblastos cuando se cultivan junto con células de la pulpa dental in vitro con capacidad de formar estructuras después de su trasplante in vivo $\left({ }^{12}\right)$.

El tejido donde se encuentran los REM abarca las áreas que están alrededor de la raíz, sobre todo las regiones supracrestales, es decir, los tejidos que contienen colágeno y que están por encima de la cresta alveolar ósea, por lo que su participación en la regeneración periodontal es cada vez más evidente. Por tanto, se deberán realizar más estudios y análisis para investigar las ubicaciones específicas y sus funciones especializadas $\left({ }^{15,16}\right)$.

\section{METODOLOGÍA}

Se realizó la búsqueda bibliográfica en Medline, SciELO y LILACS de diversos artículos usando como palabras clave "restos de Malassez" y "tejidos odontogénicos", y seleccionando las investigaciones más relevantes, las cuales fueron analizadas respecto de los siguientes tópicos teóricos.

\section{CARACTERÍSTICAS EMBRIOLÓGICAS E HISTOLÓGICAS DE LOS RESTOS EPITELIALES DE MALASSEZ}

Dentro del ligamento periodontal existe una gran cantidad de células heterógenas entre las cuales podemos encontrar poblaciones de células tanto epiteliales, endoteliales, inflamatorias, progenitoras y ectomesenquimales como fibroblastos, cementoblastos y osteoblastos, con gran capacidad de diferenciación $(17,18)$.

Entre las células epiteliales encontramos a los REM. Fue Serres fue quien las describió por primera vez considerándolas restos del órgano del esmalte. Décadas después, Legros y Magiot describieron su origen epitelial y las relacionaron con la formación de quistes y otras patologías $\left(^{2}\right)$.

Malassez, en 1885, realizó un estudio de estas células en cortes de dientes tanto transversales como longitudinales y así evidenció su presencia como una red alrededor de la raíz dentaria incluyendo el área de furcación; además, describió sus características histológicas y confirmó que se encuentran en el ligamento periodontal adulto. Por todas estas investigaciones se considera a Malassez como el investigador principal de estos restos y llevan su nombre desde entonces ${ }^{(1-3)}$. 


\section{ORIGEN}

El proceso de formación de las piezas dentarias se conoce como odontogénesis y en él se evidencian cambios morfológicos, químicos y funcionales que tienen su inicio en la sexta semana de vida intrauterina ${ }^{(1)}$. Después que la amelogénesis ha sido completada, la corona se encuentra cubierta por epitelio reducido del órgano del esmalte, y se inicia el proceso de formación radicular en el asa cervical, el cual crece en forma cónica y en dirección apical, guiando la dirección de su crecimiento, la forma, el tamaño y el número de las raíces dentarias (19-21). Esta prolongación, que se genera por la unión del epitelio interno y externo del órgano del esmalte, sin la participación del retículo estrellado, se denomina vaina radicular de Hertwig y es considerada el límite entre dos tejidos ectomesenquimales como la papila y el folículo dentales. Así mismo, es la responsable de la formación radicular. A medida que va creciendo, induce a la papila dentaria a formar los primeros odontoblastos y da inicio a la formación de la dentina radicular; este proceso hace que la vaina radicular de Hertwig pierda continuidad y nutrición por parte de la papila dental, lo que da lugar a la formación de fragmentos epiteliales llamados restos de Malassez (18, ${ }^{22}$ ). Al mismo tiempo, células ectomesenquimales del saco dentario se juntan en la superficie externa de la dentina recién formada y comienza su diferenciación en cementoblastos para formar la matriz orgánica del cemento ${ }^{(3,21-23)}$.

Por lo tanto, los REM son considerados células epiteliales o grupos de células de la vaina epitelial que sobreviven en el adulto dentro del ligamento periodontal $\left({ }^{18,24}\right)$.

\section{CARACTERÍSTICAS HISTOLÓGICAS DE LOS REM}

Histológicamente pueden ser considerados como grupos de células epiteliales ubicados dentro del ligamento periodontal con las siguientes características: son células de forma poliédrica con una relación núcleocitoplasmática muy alta, núcleo de forma irregular teñido intensamente, con presencia de heterocromatina densa, el citoplasma es pequeño delineado por un halo, mitocondrias abundantes y retículo endoplasmático rugoso poco desarrollado y la presencia de proteínas, de las cuales las más importantes son las citoqueratinas $\left({ }^{17}\right)$.

Se demostró que persisten dentro de una matriz mesenquimática en la vida posnatal $\left(^{5}\right)$. Se caracterizan también por tener en su interior agua y sustancia fundamental, las cuales transportan gracias a la ultraestructura que muchos estudios han demostrado que poseen. Nos referimos específicamente a que los REM presentan organelos de unión específicos como tonofilamentos, hemidesmosomas, desmosomas y uniones estrechas cuya función es facilitar el paso de agua e iones. Los tonofilamentos pueden estar agrupados dentro del citoplasma y se denominan tonofibrillas, las cuales no tienen contacto con el cemento $(1,3,15)$.

La distancia entre el cemento y los REM fue medida en los tres tercios de la raíz siendo en el tercio apical de 21 micrones; en el tercio medio, de 33 micrones, y en el tercio cervical, de 41 micrones, lo que indica que se encuentran más alejados cuando se aproximan a la corona $\left.{ }^{25}\right)$.

\section{CARACTERÍSTICAS FUNCIONALES Y REPARATIVAS DE LOS REM}

Entre las numerosas funciones de los REM están su participación en el mantenimiento y la regeneración del ligamento periodontal, el mantenimiento del espacio en el ligamento periodontal, la prevención de la reabsorción radicular y la anquilosis, se encargan de la homeostasis del ligamento periodontal (LPD), tienen la capacidad de curar heridas del tejido de LPD, promueven el crecimiento de terminales nerviosas, contribuyen a la diferenciación de ameloblastos, participan en la reparación y regeneración del cemento mediante una transición epitelial mesenquimal —con la posterior diferenciación en cementoblastos en la etapa inicial de formación de la raíz- $\left.{ }^{25-29}\right)$.

En cuanto al mantenimiento del espacio del LPD (que varía entre 0,20-0,40 mm), los REM son capaces de resistir situaciones donde este se ve afectado, lo que promueve la homeostasis. Esta función es desencadenada 
por la liberación de factor de crecimiento epidérmico (EGF) y las prostaglandinas en la resorción ósea $\left({ }^{30,31}\right)$.

Para la regeneración del tejido periodontal, los REM asisten en la renovación de células que sufrieron daño y registran brotes de hialinización en los lugares donde existe presión del ligamento, liberando mediadores inflamatorios, como las prostaglandinas, y proteínas del esmalte, como la amelogenina y la amelina, las cuales promueven la liberación de proteínas de la matriz ósea, osteopontina, sialoproteína y osteoprotegerina, proteínas que ayudan a la reparación y regeneración periodontal, además de la reparación del cemento. Las prostaglandinas, especialmente la E2, activan los osteoclastos para el inicio de la remodelación ósea $\left({ }^{26,29,32-34}\right)$.

Las células de los REM ayudan al depósito de tejido mineral como hueso, cemento y estructuras como las fibras de Sharpey $\left(^{5}\right)$.

Para la regeneración y reparación del cemento ayudan a la diferenciación de cementoblastos de origen mesenquimal, por lo que habrá una transformación de células epiteliales a cementoblastos. La reparación del cemento depende de la liberación de proteínas de la matriz del esmalte secretadas durante la odontogénesis $\left({ }^{9,31}\right)$.

La anquilosis es la falta de elastina como consecuencia de la pérdida de células del LPD, seguida por reabsorción radicular y el lugar que ocupaba el tejido dental es reemplazado por hueso; en este caso, la función de los REM es mantener alejado el hueso de la raíz dentaria por medio de la liberación de EGF, lo que promueve la osteoclasia. Por ejemplo, en los movimientos de ortodoncia, si son realizados de forma correcta, no se verá necrosis de los REM; por el contrario, se estimulará la secreción de estos y se evitará así la anquilosis $\left({ }^{32,35,36}\right)$.

Por otra parte, los REM ayudan a la síntesis de fibras colágenas mediante la secreción de colagenasa. Este proceso ayuda a la remodelación ósea al mantener la homeostasis y el espacio del ligamento periodontal $\left({ }^{36}\right)$.

Las células madre viven en varios tejidos y tienen la capacidad de autorrenovación y diferenciación en varios linajes. $\mathrm{Al}$ reaccionar frente a estímulos ambientales, son capaces de proliferar, ir a otros lugares y regenerar tejidos que se encuentren dañados $\left({ }^{5}\right)$.

Existen muchas investigaciones sobre la población de células madre derivadas de varios tejidos como la pulpa dental, los dientes temporarios extraídos, el ligamento periodontal, la papila apical, el folículo dental y la encía. Lamentablemente, los estudios referentes a la población de células madre a partir de los REM aún son escasos; sin embargo, se demostró que los REM son considerados células madre debido a que entran en una transformación epitelial-mesenquimática porque comparten atributos tanto fenotípicos como funcionales similares a los de las células madre mesenquimales y expresan marcadores de células madre embrionarias. Por lo tanto, pueden diferenciarse en células tanto de origen ectodérmico como mesodérmico con característica multilineal, es decir, con la capacidad de diferenciarse en osteoblastos, condrocitos, adipocitos, cementoblastos y otros; la diferenciación se puede hacer de forma in vivo como in vitro $(5,26)$.

\section{EL PAPEL DE LOS RESTOS EPITELIALES DE MALASSEZ EN LA FORMACIÓN DE PATOLOGÍAS}

El origen de quistes y tumores de los maxilares son atribuidos en gran parte a los epitelios odontogénicos embrionarios. Entre ellos encontramos los restos de Serres, que son el resultado de la descomposición de la lámina dental; los restos epiteliales de Malassez, los cuales son producto de la fragmentación de la vaina radicular de Hertwig, y los restos del epitelio reducido del esmalte. Los mencionados restos están en estado latente, pero pueden reactivarse y continuar su crecimiento, lo que da lugar a la formación de diferentes patologías $\left({ }^{37}\right)$.

Inicialmente, se produce una inflamación de origen pulpar en la región periapical o lateral radicular $\left({ }^{38,39}\right)$; posteriormente, se presenta una periodontitis apical crónica, entidad comúnmente conocida como granuloma, en la cual es común encontrar nidos de epitelio formados por los restos de Malassez con una gran capacidad de crecimiento, que forman pequeñas islas, hebras o cordones de diferentes grosores, en una 
trama de tejido compacto que no presenta licuefacción central $\left({ }^{40}\right)$. Si los restos siguen siendo estimulados por el proceso inflamatorio, seguirán proliferando hasta lograr la formación de una cavidad quística cubierta parcial o totalmente por epitelio, con contenido líquido o semilíquido $\left({ }^{41}\right)$. En ambos casos, son los estímulos inflamatorios e infecciosos los que actúan, basados en una predisposición genética, como elementos iniciales para la proliferación de estos restos epiteliales $\left({ }^{42,43}\right)$.

Histológicamente, cuando las células presentan una distribución espacial en forma de cordones reciben el nombre de granuloma apical epitelializado, y cuando no se observa esta distribución se les denomina granuloma apical simple $\left.{ }^{1,3}\right)$

El proceso de inflamación asociado a estructuras radiculares, unido a factores de crecimiento, estimulan la proliferación tridimensional de los restos epiteliales, lo que provoca una hiperplasia de los tejidos afectados por esta condición. En el caso de los quistes inflamatorios de origen dentario, el incremento del suministro de sangre es seguido por una necrosis central y, además, la pérdida de plasma circundante genera el desarrollo de estos procesos patológicos $\left({ }^{5,44}\right)$.

Para los casos del queratoquiste odontogénico, el quiste dentígero y el quiste apical, a pesar de que son diagnosticados mediante exámenes clínicos, radiológicos e histopatológicos, puede existir la posibilidad de que presenten características histológicas similares, lo que dificulta un diagnóstico definitivo. En esos casos se recurre a estudios bioquímicos como la detección de expresiones de citoqueratinas $\left({ }^{45}\right)$.

Las citoqueratinas $(\mathrm{CK})$ son parte del citoesqueleto de una célula y forman una red que se extiende desde el núcleo hasta la periferia para unirse a los desmosomas y hemidesmosomas, $\mathrm{y}$ así cumplir funciones importantes como la comunicación celular, el transporte de información y la organización citoplasmática $\left({ }^{46}\right)$. Su aparición en el proceso de formación dentaria ha sugerido también su presencia en la formación de quistes y tumores odontogénicos. Es decir, la expresión de queratina no solo permite identificar a una célula como epitelial, también permite que sea un importante auxiliar de diagnóstico para determinar la posible histogénesis de lesiones quísticas y tumorales de origen odontogénico $\left({ }^{47-50}\right)$.

Reichart y col. publicaron los resultados de una investigación para probar la teoría patogénica de los restos epiteliales de Malassez. Para ello, utilizaron técnicas de tinción convencional de quistes inflamatorios y encontraron que los restos epiteliales proliferan ante un estímulo inflamatorio, lo que tiene como consecuencia el desarrollo de un quiste radicular.

Sin embargo, en esa misma investigación, cuando se utilizó la aplicación de técnicas inmunohistoquímicas epiteliales más específicas, como las citoqueratinas monoclonales, no se logró confirmar que exista un proceso secuencial de: restos epiteliales de Malassez con la aparición de granulomas o quistes apicales $\left({ }^{39}\right)$.

Entre los quistes que se originan a partir de los REM está el quiste radicular, considerado como el más común de la cavidad oral, y que se produce después de la pérdida de la barrera biológica que protege la pulpa dental, ya sea por lesiones cariosas o trauma dental, con la consiguiente necrosis pulpar, la formación de un granuloma $y$, finalmente, la formación quística a nivel apical de un diente no vital $(43,51,52)$.

Así mismo, el crecimiento en número de los restos epiteliales es considerado como la razón principal para la formación de lesiones patológicas, siendo las más frecuentes, como ya se mencionó, los quistes inflamatorios como el periapical o el residual, el quiste periodontal, el quiste paradental y el quiste odontogénico calcificante. Está demostrado que los restos epiteliales de Malassez en estado latente durante un largo periodo pueden reanudar su crecimiento activo y dar lugar a una variedad de tumores odontogénicos como el ameloblastoma, el tumor odontogénico escamoso y el carcinoma odontogénico de células claras $\left({ }^{53}\right)$.

Entre las lesiones más importantes tenemos los siguientes:

a. Ameloblastoma. Es una lesión que se origina en los restos epiteliales odontogénicos ubicados en el cuerpo mandibular, en la cresta alveolar, en el ligamento 
periodontal, en el folículo dental o en las paredes de un quiste. Estas localizaciones indican que puede tener como origen varios tipos de restos epiteliales, entre ellos los REM ${ }^{(54)}$.

b. Tumor odontogénico de células escamosas. Está formado por una proliferación focal de epitelio odontogénico que, por lo general, se muestra como dos capas de células alrededor de la raíz de una pieza dentaria que serían los REM, y la erosión de la cresta ósea, pero no se extiende más allá del tercio apical de la raíz $\left({ }^{55}\right)$.

c. Carcinoma de células claras. Histológicamente, se ven como grandes islas de epitelio presentes en el ligamento periodontal. No hay infiltrado inflamatorio (54).

\section{CONCLUSIONES}

1. Los restos de Malassez permanecen en el ligamento periodontal durante toda la vida y cumplen funciones importantes como el mantenimiento, la regeneración y la homeostasis del ligamento periodontal, así como previenen la reabsorción radicular y la anquilosis.
2. Los REM son considerados un reservorio de células madre, las cuales tienen una gran capacidad de diferenciación en varios linajes celulares; de ahí su participación en la formación y el desarrollo de diversos aspectos regenerativos. El potencial proliferativo $\mathrm{y}$ odontogénico de estas células no se ha explotado del todo, por lo que constituyen una alternativa prometedora para la regeneración y formación de tejidos dentales.

3. El origen de algunos quistes y tumores de los maxilares son estos restos epiteliales (REM) al ser estimulados por algún proceso inflamatorio que genera su multiplicación, lo cual promueve, junto a factores de crecimiento de la región, la proliferación tridimensional de estas células y el establecimiento de la patología.

Contribución de autoría: Paola Elena Medina-Ocampo y Gustavo Fiori-Chíncaro han participado en la elaboración del artículo, la recolección de la información, la redacción y aprobación de la versión final.

Fuente de financiamiento: Autofinanciado.

Potenciales conflictos de interés: Los autores declaran no tener ningún tipo de conflicto de interés. 


\section{REFERENCIAS BIBLIOGRÁFICAS}

1. Pulitano Manisagian GE, Núñez FL, Mandalunis PM. El rol de los restos epiteliales de Malassez en el ligamento periodontal. Rev. Fac. Odontol. (B.Aires). 2012; 27: 35-46.

2. Silva BSE, Fagundes NCF, Nogueira BCL, Valladares JN, Normando D, Lima RR. Epithelial rests of Malassez: from latent cells to active participation in orthodontic movement. Dental Press J Orthod. 2017; 22 (3): 119-25. doi: 10.1590/21776709.22.3.119-125.sar

3. Rincón JC, Young WG, Bartold PM. Minirevision. Los restos epiteliales de Malassez: ¿un posible papel en la regeneracion periodontal? Univ Odontol. 2009; 28 (60): 19-28.

4. Becktor KB, Nolting D, BecktorJP, Kjaer I.Immunohistochemical localization of epithelial rests of Malassez in human periodontal membrane. Eur J Orthod. 2007; 29 (4): 350-3.

5. Xiong J, Mrozik K, Gronthos S, Bartold PM. Epithelial cell rests of Malassez contain unique stem cell populations capable of undergoing epithelial-mesenchymal transition. Stem Cells Dev. 2012; 21 (11): 2012-25. doi: 10.1089/scd.2011.0471

6. Luan X, Ito Y, Diekwisch TG. Evolution and development of Hertwig's epithelial root sheath. Dev Dyn. 2006; 235 (5): 116780. doi: 10.1002/dvdy.20674

7. Sonoyama W, Liu Y, Fang D, Yamaza T, Seo BM, Zhang C, et al. Mesenchymal stem cell-mediated functional tooth regeneration in swine. PLoS One. 2006; 1: e79. doi: 10.1371/journal. pone. 0000079

8. Foster BL, Popowics TE, Fong HK, Somerman MJ. Advances in defining regulators of cementum development and periodontal regeneration. Curr Top Dev Biol. 2007; 78: 47-126.

9. Nam H, Kim J, ParkJ, Park JC, Kim JW, Seo BM, et al. Expression profile of the stem cell markers in human Hertwig's epithelial root sheath/Epithelial rests of Malassez cells. Mol Cells. 2011; 31 (4): 355-60. doi: 10.1007/s10059-011-0045-3

10. Tsunematsu T, Fujiwara N, Yoshida M, Takayama Y, Kujiraoka S, Qi G, et al. Human odontogenic epithelial cells derived from epithelial rests of Malassez possess stem cell properties. Lab Invest. 2016; 96 (10): 1063-75.

11. Volponi AA, Pang Y, Sharpe PT. Stem cell-based biological tooth repair and regeneration. Trends Cell Biol. 2010; 20 (12): 715-22. doi: 10.1016/j.tcb.2010.09.012

12. Orsini G, Pagella P, Putignano A, Mitsiadis TA. Novel biological and technological platforms for dental clinical use. Front Physiol. 2018; 9: 1102.

13. Li J, Parada C, Chai Y. Cellular and molecular mechanisms of tooth root development. Development. 2017; 144 (3): 374-84.

14. Liu H, Yan X, Pandya M, Luan X, Diekwisch TG. Daughters of the enamel organ: development, fate, and function of the stratum intermedium, stellate reticulum, and outer enamel epithelium. Stem Cells Dev. 2016; 25 (20): 1580-90.

15. Tansriratanawong K, Ishikawa $\mathrm{H}$, Toyomura J, Sato S. Establishment and characterization of novel epithelial-like cell lines derived from human periodontal ligament tissue in vitro. Hum Cell. 2017; 30 (4): 237-48.
16. Sala-Pérez S, Marco-Molina V, Gay-Escoda C. Squamous odontogenic tumor-like proliferation in a radicular cyst: A case report. J Clin Exp Dent. 2013; 5 (5): e298-301.

17. Beertsen W, McCulloch CA, Sodek J. The periodontal ligament: a unique, multifunctional connective tissue. Periodontol. 2000. 1997; 13: 20-40. doi: 10.1111/j.1600-0757.1997.tb00094.x

18. Diekwisch TG. The developmental biology of cementum. Int J Dev Biol. 2001; 45 (5-6): 695-706.

19. Yamamoto T, Hasegawa T, Yamamoto T, Hongo H, Amizuka N. Histology of human cementum: Its structure, function, and development. Jpn Dent Sci Rev. 2016; 52 (3): 63-74.

20. Huang X, Bringas P, Jr., Slavkin HC, Chai Y. Fate of HERS during tooth root development. Dev Biol. 2009; 334 (1): 22-30.

21. Sakano M, Otsu K, Fujiwara N, Fukumoto S, Yamada A, Harada $\mathrm{H}$. Cell dynamics in cervical loop epithelium during transition from crown to root: implications for Hertwig's epithelial root sheath formation. J Periodontal Res. 2013; 48 (2): 262-7.

22. Vatanpour M, Zarei M, Javidi M, Shirazian S. A new approach to root formation. Iran Endod J. 2008; 3 (2): 42-3.

23. Yamamoto T, Yamamoto T, Yamada T, Hasegawa T, Hongo $H$, Oda K, et al. Hertwig's epithelial root sheath cell behavior during initial acellular cementogenesis in rat molars. Histochem Cell Biol. 2014; 142 (5): 489-96.

24. Cho MI, Garant PR. Development and general structure of the periodontium. Periodontol 2000. 2000; 24: 9-27.

25. Rincon JC, Young WG, Bartold PM. The epithelial cell rests of Malassez--a role in periodontal regeneration? J Periodontal Res. 2006; 41 (4): 245-52.

26. Tadokoro O, Maeda T, Heyeraas KJ, Vandevska-Radunovic V, Kozawa Y, Hals Kvinnsland I. Merkel-like cells in Malassez epithelium in the periodontal ligament of cats: an immunohistochemical, confocal-laser scanning and immuno electron-microscopic investigation. J Periodontal Res. 2002; 37 (6): 456-63.

27. Liu F, Abiko Y, Nishimura M, Kusano K, Shi S, Kaku T. Expression of inflammatory cytokines and beta-defensin 1 mRNAs in porcine epithelial rests of Malassez in vitro. Med Electron Microsc. 2001; 34 (3): 174-8.

28. Guajardo G, Okamoto Y, Gogen H, Shanfeld JL, Dobeck $\mathrm{J}$, Herring $\mathrm{AH}$, et al. Immunohistochemical localization of epidermal growth factor in cat paradental tissues during tooth movement. Am J Orthod Dentofacial Orthop. 2000; 118 (2): 210-9. doi: 10.1067/mod.2000.104097

29. Xiong J, Gronthos S, Bartold PM. Role of the epithelial cell rests of Malassez in the development, maintenance and regeneration of periodontal ligament tissues. Periodontol 2000. 2013; 63 (1): 217-33. doi: 10.1111/prd.12023

30. Keinan D, Cohen RE. The significance of epithelial rests of Malassez in the periodontal ligament. J Endod. 2013; 39 (5): 582-7. doi: 10.1016/j.joen.2013.01.004 
31. Koshihara T, Matsuzaka K, Sato T, Inoue T. Effect of stretching force on the cells of epithelial rests of malassez in vitro. Int J Dent. 2010; 2010: 458408.

32. Hasegawa N, Kawaguchi H, Ogawa T, Uchida T, Kurihara H. Immunohistochemical characteristics of epithelial cell rests of Malassez during cementum repair. J Periodontal Res. 2003; 38 (1): 51-6. doi: 10.1034/j.1600-0765.2003.01636.x

33. Mutoh N, Nakatomi M, Ida-Yonemochi H, Nakagawa E, TaniIshii N, Ohshima H. Responses of BrdU label-retaining dental pulp cells to allogenic tooth transplantation into mouse maxilla. Histochem Cell Biol. 2011; 136 (6): 649-61.

34. Consolaro A, Consolaro M. Funciones de ERM,EGF y movimiento ortodontico o porque el movimiento ostodontico no causa anquilosis alveolodental. Prensa dental J Orthod 2010; 2 : 24-32.

35. Kale S, Kocadereli I, Atilla P, Asan E. Comparison of the effects of 1,25 dihydroxycholecalciferol and prostaglandin E2 on orthodontic tooth movement. Am J Orthod Dentofacial Orthop. 2004; 125 (5): 607-14. doi: 10.1016/j.ajodo.2003.06.002

36. von Bohl M, Kuijpers-Jagtman AM. Hyalinizationduring orthodontic tooth movement: a systematic review on tissue reactions. Eur J Orthod. 2009; 31 (1): 30-6.

37. Wang Y, Lv L, Yu X, Zhang T, Li S. The characteristics of epithelial cell rests of Malassez during tooth eruption of development mice. J Mol Histol. 2014; 45 (1): 1-10.

38. Ide F, Obara K, Yamada H, Mishima K, Saito I, Horie N, et al. Hamartomatous proliferations of odontogenic epithelium within the jaws: a potential histogenetic source of intraosseous epithelial odontogenic tumors. J Oral Pathol Med. 2007; 36 (4): 229-35.

39. Reichart PA, Philipsen HP. Odontogenic tumors and allied lesions. London: Quintessence Publishing; 2004.

40. Ide F, Obara K, Mishima K, Saito I, Horie N, Shimoyama T, et al. Peripheral odontogenic tumor: a clinicopathologic study of 30 cases. General features and hamartomatous lesions. J Oral Pathol Med. 2005; 34 (9): 552-7. doi: 10.1111/j.16000714.2005.00355.x

41. Leon JE, Mata GM, Fregnani ER, Carlos-Bregni R, de Almeida OP, Mosqueda-Taylor A, et al. Clinicopathological and immunohistochemical study of 39 cases of Adenomatoid Odontogenic Tumour: a multicentric study. Oral Oncol. 2005; 41 (8): 835-42. doi: 10.1016/j.oraloncology.2005.04.008

42. Yoon HJ, Jo BC, Shin WJ, Cho YA, Lee JI, Hong SP, et al. Comparative immunohistochemical study of ameloblastoma and ameloblastic carcinoma. Oral Surg Oral Med Oral Pathol Oral Radiol Endod. 2011; 112 (6): 767-76.
43. Neville BW, Damm DD, Allen CM, Bouquot JE. Enfermedades de la pulpa y del periapice. Patologia oral y maxilofacial 2004; 3 : 105-29.

44. Takahashi K, Shimonishi M, Wang R, Watanabe H, Kikuchi $\mathrm{M}$. Epithelial-mesenchymal interactions induce enamel matrix proteins and proteases in the epithelial cells of the rests of Malassez in vitro. Eur J Oral Sci. 2012; 120 (6): 475-83.

45. Talic NF, Evans CA, Daniel JC, Zaki AE. Proliferation of epithelial rests of Malassez during experimental tooth movement. Am J Orthod Dentofacial Orthop. 2003; 123 (5): 527-33.

46. Philipsen HP, Reichart PA. The development and fate of epithelial residues after completion of the human odontogenesis with special reference to the origins of epithelial odontogenic neoplasms, hamartomas and cysts. Oral Biosci Med. 2004; 1 : 171-9.

47. Bilodeau EA, Collins BM. Odontogenic cysts and neoplasms. Surg Pathol Clin. 2017; 10 (1): 177-222.

48. Stoll C, Stollenwerk C, Riediger D, Mittermayer C, Alfer J. Cytokeratin expression patterns for distinction of odontogenic keratocysts from dentigerous and radicular cysts. J Oral Pathol Med. 2005; 34 (9): 558-64.

49. Chu PG, Weiss LM. Keratin expression in human tissues and neoplasms. Histopathology. 2002; 40 (5): 403-39.

50. Crivelini MM, de Araujo VC, de Sousa SO, de Araujo NS. Cytokeratins in epithelia of odontogenic neoplasms. Oral Dis. 2003; 9 (1): 1-6. doi: 10.1034/j.1601-0825.2003.00861.x

51. Ricucci D, Pascon EA, Ford TR, Langeland K. Epithelium and bacteria in periapical lesions. Oral Surg Oral Med Oral Pathol Oral Radiol Endod. 2006; 101 (2): 239-49. doi: 10.1016/j. tripleo.2005.03.038

52. Haku K, Muramatsu T, Hara A, Kikuchi A, Hashimoto S, Inoue T, et al. Epithelial cell rests of Malassez modulate cell proliferation, differentiation and apoptosis via gap junctional communication under mechanical stretching in vitro. Bull Tokyo Dent Coll. 2011; 52 (4): 173-82. doi: 10.2209/tdcpublication.52.173

53. Rosenberg PA, Frisbie J, Lee J, Lee K, Frommer H, Kottal S, et al. Evaluation of pathologists (histopathology) and radiologists (cone beam computed tomography) differentiating radicular cysts from granulomas. J Endod. 2010; 36 (3): 423-8.

54. Carrillo C, Penarrocha M, Ortega B, Marti E, Bagan JV, Vera F. Correlation of radiographic size and the presence of radiopaque lamina with histological findings in 70 periapical lesions. J Oral Maxillofac Surg. 2008; 66 (8): 1600-5.

55. Tsuneki M, Yamazaki M, Cheng J, Maruyama S, Kobayashi T, Saku T. Combined immunohistochemistry for the differential diagnosis of cystic jaw lesions: its practical use in surgical pathology. Histopathology. 2010; 57 (6): 806-13. 